\title{
Nanoscale analysis of electrical junctions in InGaP nanowires grown by template-assisted selective epitaxy
}

Cite as: Appl. Phys. Lett. 114, 103101 (2019); doi: 10.1063/1.5085405

Submitted: 12 December 2018 - Accepted: 12 February 2019 .

Published Online: 11 March 2019
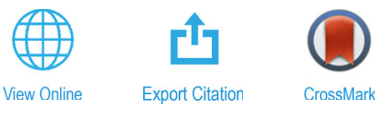

\author{
V. Piazza, ${ }^{1, a)}$ (D) S. Wirths, ${ }^{2}$ N. Bologna, ${ }^{2,3}$ A. A. Ahmed, ${ }^{1}$ F. Bayle, ${ }^{7}$ H. Schmid, ${ }^{2}$ (D) F. Julien, ${ }^{1}$ and M. Tchernycheva
}

\begin{abstract}
AFFILIATIONS
${ }^{7}$ Centre de Nanosciences et de Nanotechnologies (C2N), UMR 9001 CNRS, Univ. Paris Sud, Univ. Paris-Saclay, 8 Avenue de la Vauve, 91120 Palaiseau, France

${ }^{2}$ IBM Research - Zurich, Säumerstrasse 4, 8803 Rüschlikon, Switzerland

${ }^{3}$ Electron Microscopy Center, EMPA, Swiss Federal Laboratories for Materials Science and Technology, 8600 Dübendorf, Switzerland

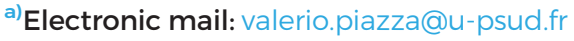

\begin{abstract}
We report the analysis of the electrical properties of $\operatorname{In}_{x-1} \mathrm{Ga}_{\mathrm{x}} \mathrm{P}$ nanowires (NWs) grown by template-assisted selective epitaxy. The individual NW properties are investigated by means of electron beam induced current microscopy (EBIC) and current-voltage curves acquired on single nano-objects. First, a set of samples containing n-doped InGaP NWs grown on a p-doped Si substrate are investigated. The electrical activity of the hetero-junction between the NWs and the substrate is demonstrated and the material parameters are analyzed, namely, the $\mathrm{n}$-doping level is determined in relation to the dopant flow used during the growth. These results were used to design and elaborate InGaP NWs containing a p-n homo-junction. The electrical activity of the homo-junction is evidenced using EBIC mapping on single NWs, and material parameters (namely, the doping and the minority carrier diffusion lengths) for the p- and n-doped InGaP segments are estimated. Finally, the first proof of a photovoltaic effect from the NW homo-junctions is obtained by photocurrent measurements of a contacted NW array under white light irradiation.
\end{abstract}

Published under license by AIP Publishing. https://oi.org/10.1063/1.5085405

Multi-junction solar cells represent one possible solution to overcome the Shockley-Queisser thermodynamic limit. In these devices, different materials absorbing in different spectral ranges are stacked, thus decreasing transmission and thermalization losses. ${ }^{1-3}$ This approach led to the fabrication of three- and four-junction solar cells holding the actual photovoltaic conversion efficiency record of $44.7 \%$ and $46.1 \% \cdot{ }^{2,4,5}$ Nevertheless, the spreading of multi-junction solar cells in the market is hindered by their high costs due to the use of expensive III-V materials and costly substrates.

Nanowire (NW)-based tandem solar cells have been proposed to address the aforementioned issues thanks to the reduced amount of III-V materials needed for efficient absorption and the possibility to grow them epitaxially on low-cost $\mathrm{Si}$ substrates. It has been demonstrated that nanowires (NWs) offer the possibility to obtain a highquality material on lattice-mismatched substrates due to the stress relaxation through the lateral surface. ${ }^{6}$ Furthermore, the optical absorption in NWs is enhanced due to their small reflectance and enhanced light diffusion in the NW array. ${ }^{7,8}$ Based on these advantages, a two-junction NW on a Si tandem solar cell has been proposed. ${ }^{9}$ It has been shown that an efficiency around $40 \%$ under concentration can be achieved for the optimal top cell bandgap of 1.7 $\mathrm{eV} .^{9,10}$ This bandgap value can be reached using III-V ternary alloys. Therefore, the growth of ternary III-V NWs on Si is of great interest in view of fabricating III/V on Si tandem solar cells.

Recently, the template-assisted selective epitaxy (TASE) technique has been proposed as a method to monolithically grow III-V NWs on $\mathrm{Si}(100)^{11}$ and (111). ${ }^{12,13}$ TASE enables the growth of nanostructures inside a $\mathrm{SiO}_{2}$ nanotube template, which imposes the NW positions, diameters, and verticality, resulting in a better-controlled process than other NW elaboration techniques. In addition, without the presence of a catalyst particle to promote the $1 \mathrm{D}$ growth, abrupt heterostructures can be formed and parasitic radial growth phenomena are suppressed. ${ }^{14}$ The use of TASE has been recently extended to the growth of ternary InGaP NWs and the effect of p- and n-dopants on the crystal structure, composition of the alloy, and optical properties has been investigated. ${ }^{15}$ In the present work, we report the first 

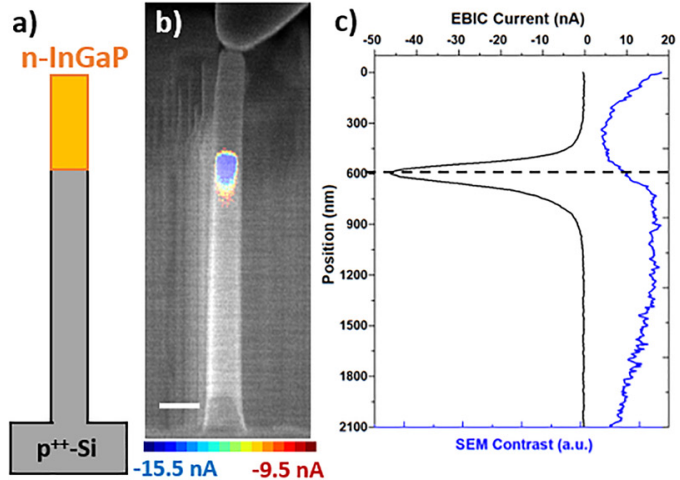

d)

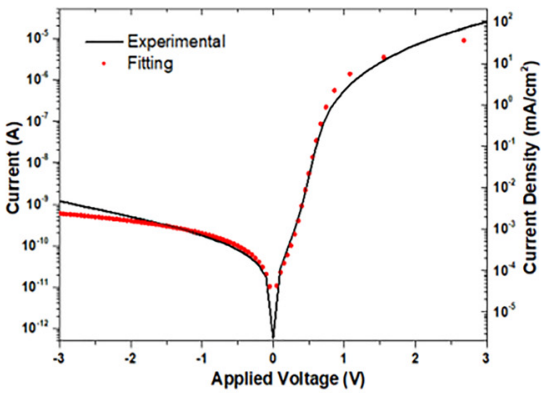

FIG. 1. (a) Schematic of the $n-\ln G a P /$ $\mathrm{p}^{++}$-Si NWs; (b) secondary electron (SE) image of a single NW overlapped with the corresponding EBIC signal at $V_{\text {bias }}$ $=-3 \mathrm{~V}$ (temperature color current scale is reported in the inset); the scale bar corresponds to $250 \mathrm{~nm}$. (c) EBIC profile (black cure) extracted along the NW axis in panel (b) and corresponding SE profile (blue curve); (d) single NW I(V) and J(V) characteristics in the dark on a semilogarithmic scale (black curve) and the corresponding fitting with a single diode model (red dots)

investigation of the electrical properties at the nanoscale of these InGaP NWs by electron beam induced current microscopy (EBIC) and $\mathrm{I}(\mathrm{V})$ measurements performed on single nanostructures. Two different sets of samples were analyzed: the first contains homogeneously n-doped InGaP NWs to examine the n-doping using a heterojunction with the p-Si substrate, while the second consists of InGaP NWs containing a p-n homo-junction and aims to study the nanoscale charge collection properties of these structures.

The investigated samples were grown by metal-organic chemical vapor deposition (MOCVD) on a $\mathrm{p}^{++}$-doped $\mathrm{Si}(111)$ substrate using the TASE approach with a $\mathrm{SiO}_{2}$ tube template. ${ }^{11,16} \mathrm{In}_{\mathrm{x}-1} \mathrm{Ga}_{\mathrm{x}} \mathrm{P}$ (with a nominal composition $\mathrm{x}=0.3) \mathrm{NWs}$ were grown at $650{ }^{\circ} \mathrm{C}$ and 60 Torr, using trimethylindium (TMIn), trimethylgallium (TMGa), tertiarybutylphosphine (TBP), and diethylzinc (DEZn) and disilane $\left(\mathrm{Si}_{2} \mathrm{H}_{6}\right)$ as dopants. The V/III molar ratio was fixed to 32 and TMGa/ (TMGa + TMIn) to 0.43. For n-type doping, the Si molar ratio (Si/ TMIn + TMGa) was varied from $2.5 \times 10^{-4}$ to $2.4 \times 10^{-3} \mathrm{sccm}$ (indicated as $\mathrm{Si}_{2} \mathrm{H}_{6}$ flow from 0.25 to 2.4 in the following). For the p-type doping, the (DEZn/TMIn + TMGa) ratio was set to 1.6. A thorough structural and compositional characterization can be found in Ref. 15.

Single NWs are investigated by EBIC microscopy to visualize the electrical activity of the junction. The EBIC setup consists of a Hitachi SU-8000 SEM where Kleindiek PS4 nano-manipulators equipped with $100 \mathrm{~nm}$ radius tungsten tips ensure the electrical contact to single nanostructures. In EBIC microscopy, electron-hole pairs are locally generated by the electron beam. The excess charges created close to internal field regions can be separated and collected producing a measurable current. ${ }^{17-19}$ EBIC measurements were performed at a $10 \mathrm{kV}$ acceleration voltage and a $131 \mathrm{pA}$ injected current applying a bias ranging from $-4 \mathrm{~V}$ to $+4 \mathrm{~V}$ through a Stanford SR560 preamplifier controlled by a Gatan DigiScan system. More details about the set-up can be found in Refs. 19 and 20. I(V) measurements were performed on single NWs using a Keithley 2636 source-meter with a noise below $0.3 \mathrm{pA}$. For all the reported measurements, the top of the NWs was connected to the negative pole of the source-meter while the Si substrate was connected to the positive pole.

First, the NWs containing an n-doped InGaP segment on top of a $\mathrm{p}^{++}$-Si wafer were investigated [Fig. 1(a)]. The EBIC maps on single NWs show that the $\mathrm{p}^{++}-\mathrm{Si} / \mathrm{n}-\mathrm{InGaP}$ interface is electrically active under reverse bias [Fig. 1(b)]. In these conditions, a localized negative induced current is collected coherently with the direction of the field in the p-n hetero-junction. As expected, no signal is detected when a forward bias is applied.

The different secondary electron (SE) contrast of the InGaP and Si segments shown in Fig. 1(b) (respectively, darker and brighter) allows us to estimate the length of the n-InGaP segment. By plotting the SE contrast profile along the axis of the NW [blue curve in Fig. 1(b)], this segment is estimated to be around $580 \pm 50 \mathrm{~nm}$. This position coincides with the position of the peak in the collected induced current [black curve in Fig. 1(c)] proving the electrical activity of this interface. The electrical behavior of single NWs is also investigated by measuring the I(V) characteristics [Fig. 1(d)]. In agreement with EBIC observations, a rectifying behavior is evidenced. By fitting the curve with a single diode model, the junction's saturation current is estimated to be around $0.5 \mathrm{pA}$ and the ideality factor around 2.1, while series and shunt resistances are estimated to be around $400 \mathrm{k} \Omega$ and
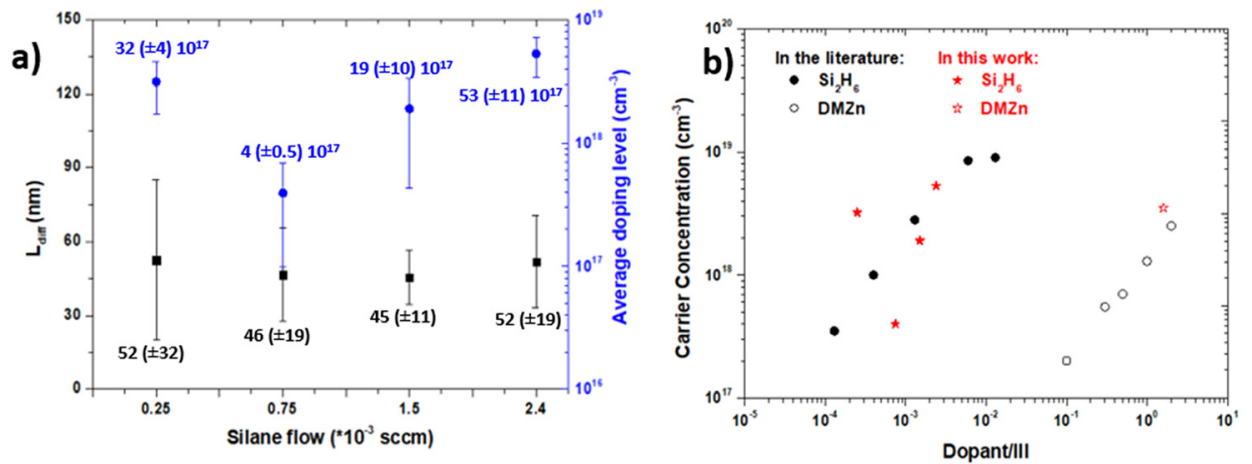

FIG. 2. (a) Hole diffusion length values (black) and average doping level (blue) estimated for the different samples. The error bars reflect the dispersion over the investigated NWs; (b) carrier concentration vs dopant flow graph, comparing the data from Ref. 26 (in black) and the values estimated in this work (in red). 
$4 \mathrm{G} \Omega$, respectively [red dots in Fig. 1(d)]. Along with the high shunt resistance value, the $\mathrm{I}(\mathrm{V})$ curve shows that the leakage current in reverse bias is negligible compared to the forward current.

A set of samples was grown under different dopant flows $\left(\mathrm{Si}_{2} \mathrm{H}_{6}\right.$ flow from 0.25 to 2.4 ) to analyze the n-doping level in the ternary alloy combining EBIC microscopy and voltage-induced SE contrast analysis (VSEC) as done by Tchoulfian et al. on radial GaN NWs. ${ }^{21}$ The SE profile can be used to localize the junction position ${ }^{22}$ since the SE electron yield is sensitive to the material properties ${ }^{23}$ and doping type. ${ }^{24}$ At the same time, EBIC profiles allow us to estimate the minority carrier diffusion length $\left(\mathrm{L}_{\mathrm{diff}}\right)^{18}$ and the extension of the space charge region (SCR) ${ }^{25}$ at the nanoscale. Combining the two techniques, it is possible to measure separately the SCR extension in the p-doped and $\mathrm{n}$-doped parts of the junction which depends on the corresponding doping levels. Further details can be found in Ref. 21. To estimate the doping level, we assume that the carrier motion in the SCR is governed by a pure drift, while outside the SCR, the transport of the generated carriers is ruled by diffusion. The details about the model can be found in the supplementary material.

The results are shown in Fig. 2(a) which reports the hole diffusion length values $\left(\mathrm{L}_{\mathrm{diff}}\right)$ and the average doping levels for each sample. The error bars reflect the dispersion of values over 12 NWs. The diffusion length is around $\sim 50 \mathrm{~nm}$ for all the samples. Taking into account the excellent crystal quality reported by Bologna et al. on similar nanoobjects, ${ }^{15}$ it is likely that the diffusion length is dominated by surface recombination and is insensitive to the increase in the dopant concentration. On the other side, this result confirms the reproducibility of the reported estimation method.

The average doping level is estimated between $10^{17}$ and $10^{19} \mathrm{~cm}^{-3}$ for the different samples. Fluctuations are found from NW to NW as reflected in the standard deviation (given in parentheses in the figure), which shows that the statistical error is $12 \%-20 \%$. The variation of the estimated doping level in different samples shows that when the dopant flow is increased from 0.75 to 2.4 , the donor concentration increases from $3.9 \times 10^{17} \mathrm{~cm}^{-3}$ to $5.3 \times 10^{18} \mathrm{~cm}^{-3}$, with a notable exception of the lowest flow of 0.25 , which deviates from the expected trend and needs further investigation. However, the doping level estimated for a dopant flow higher than 0.25 is coherent with carrier concentration values reported in the literature for the $\operatorname{In}_{0.65} \mathrm{Ga}_{0.35} \mathrm{P}$ epitaxial layer, ${ }^{26}$ as shown in Fig. 2(b). It is worth noticing that the variation of the doping level with the dopant flow exhibits a different slope than the one reported for $2 \mathrm{D}$ layers. ${ }^{26}$ Different explanations can be proposed, such as a different dopant incorporation in TASE NWs compared to thin films.

Next, TASE-grown InGaP nanowire $\mathrm{p}-\mathrm{n}$ junctions on the $\mathrm{p}^{++}-\mathrm{Si}$ substrate were investigated [schematic in Fig. 3(a)]. The highest silane flow of the doping series (2.4) was used for the doping of the n-InGaP segment. EBIC maps revealed different electrical signals under positive and negative external biases. At a positive bias, the $\mathrm{p}-\mathrm{n}$ InGaP homojunction is electrically active [Fig. 3(b)] and the current is coherent with the expected direction for the built-in field (i.e., a positive induced current as indicated by the color-coded scale). This observation constitutes the first evidence of charge collection in ternary alloy p-n junction NWs grown by TASE. Under negative bias, the $\mathrm{p}-\mathrm{n}$ homojunction is flattened and other rectifying elements with opposite orientation are revealed [Fig. 3(c)], namely, two signals are observed. The more intense signal is located at the contact point between the
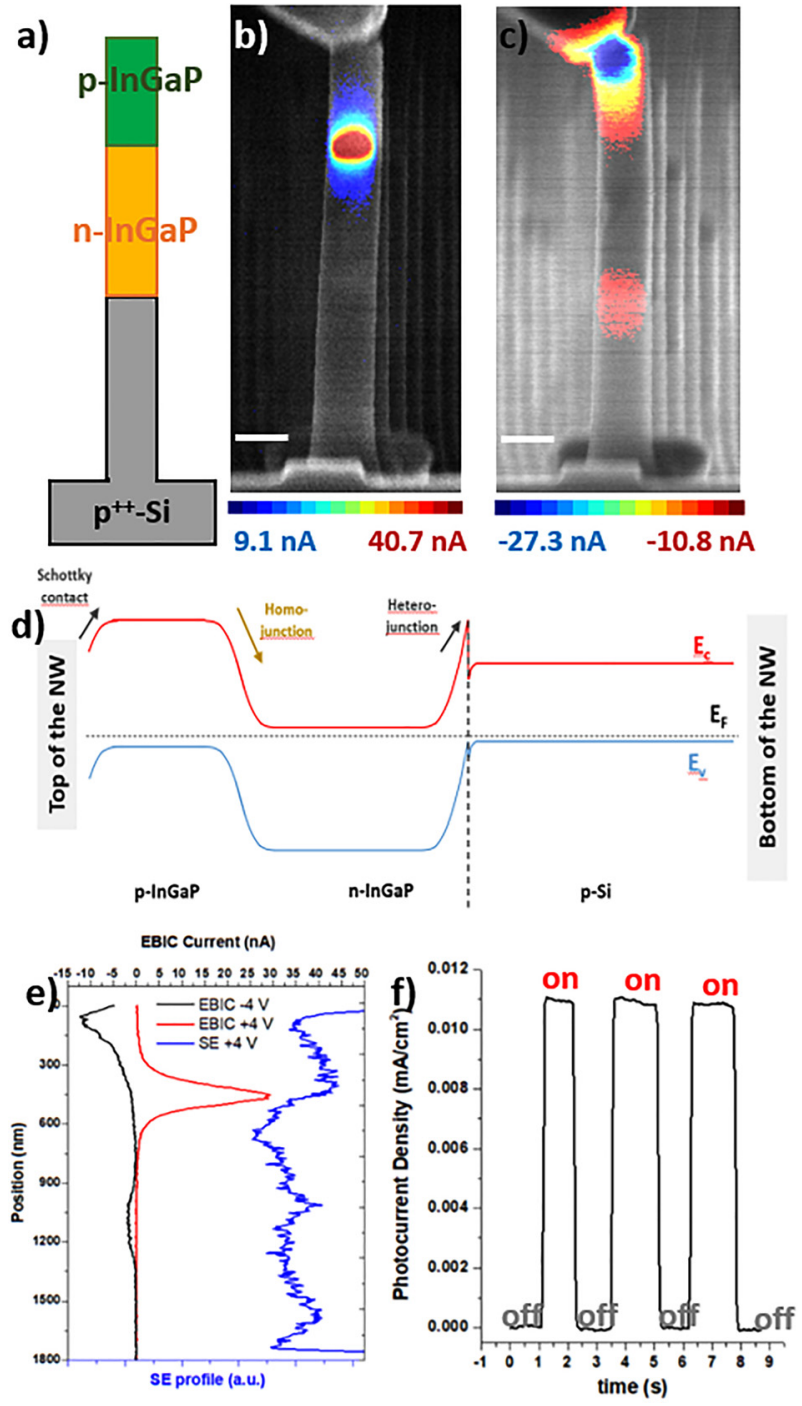

FIG. 3. (a) Schematic of a NW containing an InGaP p-n junction; SE images of a single NW superimposed with the corresponding EBIC signals at $\mathrm{V}_{\text {bias }}=4 \mathrm{~V}(\mathrm{~b})$ and $V_{\text {bias }}=-4 \mathrm{~V}$ (c) (current is traced using a temperature color code, and the scale is given below each panel). The scale bars correspond to $200 \mathrm{~nm}$; (d) axial band diagram simulated with NextNano software, highlighting the local band bending in individual NWs consistently with the EBIC maps in panels (b) and (c); (e) $\mathrm{EBIC}$ profile at $\mathrm{V}_{\text {bias }}=4 \mathrm{~V}$ (in red) and $\mathrm{V}_{\text {bias }}=-4 \mathrm{~V}$ (in black) extracted along the main axis of the NW in panels (b) and (c). The blue curve representing the SE profile at $\mathrm{V}_{\text {bias }}=4 \mathrm{~V}$ highlights the contrast variation corresponding to the EBIC peak; (f) photocurrent density produced by the NW array alternating dark conditions and white light illumination.

nanomanipulator and the NW. It is related to the presence of a Schottky barrier between the $\mathrm{p}-\mathrm{InGaP}$ and the tungsten tip.

A weaker signal of the same negative sign is observed in the lower NW part at the interface between $\mathrm{Si}$ and $\mathrm{InGaP}$. This signal arises from the heterojunction $\mathrm{p}^{++}-\mathrm{Si} / \mathrm{n}-\mathrm{InGaP}$ as described in the previous set of samples. At zero bias, no measurable signal could be found, which is due to the presence of the above-described multiple diodes 
with opposite directions. By simulating the axial band profile with the NextNano software, the rectifying interfaces in individual NWs were identified, consistent with the EBIC maps reported in panels b and $c$ [Fig. 3(d)].

A comparison of the EBIC and SE profiles [Fig. 3(e)] shows that the EBIC peak corresponds to a sharp decrease in the SE contrast, which is associated with the presence of the $p$ - $n$ homojunction. Through the analysis of the profile, doping level and $\mathrm{L}_{\text {diff }}$ are estimated in both the $\mathrm{n}$ - and p-doped segments. In the $\mathrm{n}$-InGaP segment, the doping level is estimated to be around $3.4 \times 10^{18} \mathrm{~cm}^{-3}$ and the hole diffusion length is found to be $44 \pm 10 \mathrm{~nm}$ : these results are coherent with the values estimated in the previous series of samples. In the $\mathrm{p}$-InGaP segment, the doping level is estimated to be around $3.5 \times 10^{18} \mathrm{~cm}^{-3}$ and the electron diffusion length is around $52 \pm 10 \mathrm{~nm}$.

The positive induced current produced by the $\mathrm{p}-\mathrm{n}$ InGaP junction ( $\sim 28 \mathrm{nA}$ at the peak) is more pronounced than the opposite current from the InGaP/Si heterojunction $(\sim 2.5 \mathrm{nA}$ at the peak). Nevertheless, these two diodes are connected in series and the overall activity of the NW results from a competition between the two opposite currents (and also the Schottky-related diode at the NW/nanomanipulator contact). This information could be potentially obtained from the EBIC maps acquired without bias (not reported), but in the present case, these maps show no current collection due to the presence of a Schottky barrier at the contact interface.

To study the photocurrent generation properties, the response of the NW array under white light (halogen lamp, $31.5 \mathrm{~mW} / \mathrm{cm}^{2}$ power density) was analyzed in short-circuit conditions. For this purpose, NWs are encapsulated in hydrogen silsesquioxane (HSQ) baked at $200{ }^{\circ} \mathrm{C}$ for $60 \mathrm{~min}$ and an indium tin oxide (ITO) contact is sputtered on top to connect a macroscopic amount (about $10^{7}$ ) of NWs in parallel. The photocurrent was measured alternating light and dark conditions and recording the current temporal trace $\mathrm{I}(\mathrm{t})$. The results in Fig. 3(e) ("on" and "off" refer to the lamp irradiation) show that a positive current is generated under illumination coherently with the orientation of the $\mathrm{p}-\mathrm{n}$ InGaP homojunction. This observation provides the first evidence of a photovoltaic effect in InGaP NWs grown by TASE.

In conclusion, in this work, we performed the study of electrical properties of InGaP NWs grown by a TASE approach. First, the electrical activity of the $\mathrm{n}-\mathrm{InGaP} / \mathrm{p}-\mathrm{Si}$ hetero-junction was probed. Quantitative analyses of the generation profiles allow us to estimate the doping in $\mathrm{n}$-InGaP NWs and to evaluate the hole diffusion length. Next, InGaP NW p-n homo-junctions were elaborated by TASE. The EBIC mapping of these structures allowed us to demonstrate the photovoltaic activity of the ternary homo-junction and to estimate the minority carrier diffusion lengths in the $\mathrm{n}$ - and $\mathrm{p}$-InGaP segments. The current generated by the homo-junction dominates over the current induced at the InGaP/Si interface. The photovoltaic activity of the homo-junction is confirmed by the analyses of short-circuit current in NW arrays under white light illumination. The analyzed NW structures represent the fundamental building block of a future InGaP-onSi monolithically integrated tandem solar cell.

See supplementary material for the details on the model used for the doping level estimation.
This work was financially supported by the European Union's Horizon 2020 research and innovation program "Nano-Tandem" under Grant Agreement No. 641023.

\section{REFERENCES}

${ }^{1}$ S. Essig, C. Allebé, T. Remo, J. F. Geisz, M. A. Steiner, K. Horowitz, L. Barraud, J. S. Ward, M. Schnabel, A. Descoeudres, D. L. Young, M. Woodhouse, M. Despeisse, C. Ballif, and A. Tamboli, Nat. Energy 2, 17144 (2017).

${ }^{2}$ F. Dimroth, M. Grave, P. Beutel, U. Fiedeler, C. Karcher, T. N. D. Tibbits, E. Oliva, G. Siefer, M. Schachtner, A. Wekkeli, A. Bett, R. Krause, M. Piccin, N. Blanc, C. Drazek, E. Guiot, B. Ghyselen, T. Salvetat, A. Tauzin, and K. Schwarzburg, Prog. Photovoltaics Res. Appl. 22, 277 (2014).

${ }^{3}$ K. A. Bertness, S. R. Kurtz, D. J. Friedman, A. E. Kibbler, C. Kramer, and J. M. Olson, Appl. Phys. Lett. 65, 989 (1994).

${ }^{4}$ M. A. Green, K. Emery, Y. Hishikawa, W. Warta, E. D. Dunlop, D. H. Levi, and A. W. Y. Ho-Baillie, Prog. Photovoltaics Res. Appl. 25, 3 (2017).

${ }^{5}$ E. Guiot, A. Drouin, C. Charles-Alfred, C. Drazek, A. de Buttet, A. Tauzin, T. Tibbits, P. Beutel, C. Karcher, E. Oliva, G. Siefer, and F. Dimroth, in CS ManTech Conference (Miami, 2016).

${ }^{6}$ K. L. Kavanagh, Semicond. Sci. Technol. 25, 024006 (2010).

${ }^{7}$ P. Krogstrup, H. I. Jorgenses, M. Heiss, O. Demichel, J. V. Holm, M. Aagesen, J. Nygard, and A. Fontcuberta i Morral, Nat. Photonics 7, 306 (2013).

${ }^{8}$ T. Stelzner, M. Pietsch, G. Andrä, F. Falk, E. Ose, and S. Christiansen, Nanotechnology 19, 295203 (2008).

${ }^{9}$ R. R. LaPierre, J. Appl. Phys. 110, 014310 (2011).

${ }^{10}$ N. Huang, C. Lin, and M. L. Povinelli, J. Appl, Phys. 112, 064321 (2012).

${ }^{11}$ M. Borg, H. Schmid, K. E. Moselund, G. Signorello, L. Gignac, J. Bruley, C. Breslin, P. Das Kanungo, P. Werner, and H. Riel, Nano Lett. 14, 1914 (2014).

${ }^{12}$ H. Schmid, M. Borg, K. Moselund, L. Gignac, C. M. Breslin, J. Bruley, D. Cutaia, and H. Riel, Appl. Phys. Lett. 106, 233101 (2015).

${ }^{13}$ M. Borg, H. Schmid, K. E. Moselund, D. Cutaia, and H. Riel, J. Appl. Phys. 117, 144303 (2015).

${ }^{14}$ M. Knoedler, N. Bologna, H. Schmid, M. Borg, K. E. Moselund, S. Wirths, M. D. Rossell, and H. Riel, Cryst. Growth Des. 17, 6297 (2017).

${ }^{15}$ N. Bologna, S. Wirths, L. Francaviglia, M. Campanini, H. Schmid, V. Theofylaktopoulos, K. E. Moselund, A. Fontcuberta i Morral, R. Erni, H. Riel, and M. D. Rossell, ACS Appl. Mater. Interfaces 10(38), 32588-32596 (2018).

${ }^{16}$ P. D. Kanungo, H. Schmid, M. T. Björk, L. M. Gignac, C. Breslin, J. Bruley, C. D. Bessire, and H. Riel, Nanotechnology 24, 225304 (2013).

${ }^{17}$ H. J. Leamy, J. Appl. Phys. 53, R51 (1982).

${ }^{18}$ E. B. Yakimov, S. S. Borisov, and S. I. Zaitsev, Phys. Tech. Poluprovodiekov 41, 426-428 (2007).

${ }^{19}$ A. S. Togonal, M. Foldyna, W. Chen, J. X. Wang, V. Neplokh, M. Tchernycheva, J. Nassar, P. Roca i Cabarrocas, and Rusli, J. Phys. Chem. C 120, 2962 (2015).

${ }^{20}$ M. Tchernycheva, V. Neplokh, H. Zhang, P. Lavenus, L. Rigutti, F. Bayle, F. H. Julien, A. Babichev, G. Jacopin, L. Largeau, R. Ciechonski, G. Vescovi, and O. Kryliouk, Nanoscale 7, 11692 (2015).

${ }^{21}$ P. Tchoulfian, F. Donatini, F. Levy, A. Dussaigne, P. Ferret, and J. Pernot, Nano Lett. 14, 3491-3498 (2014).

${ }^{22}$ P. Narchi, V. Neplokh, V. Piazza, T. Bearda, F. Bayle, M. Foldyna, C. Toccafondi, P. Prod'homme, M. Tchernycheva, and P. R. i Cabarrocas, Sol. Energy Mater. Sol. Cells 161, 263 (2017)

${ }^{23}$ H. Seiler, J. Appl. Phys. 54, R1 (1983).

${ }^{24}$ C. P. Sealy, M. R. Castell, and P. R. Wilshaw, J. Electron Microsc. (Tokyo) 49, 311-321 (2000).

${ }^{25}$ G. Otnes, E. Barrigón, C. Sundvall, K. E. Svensson, M. Heurlin, G. Siefer, L. Samuelson, I. Åberg, and M. T. Borgström, Nano Lett. 18, 3038 (2018).

${ }^{26}$ J. Lin, M. Wu, M. Jou, C. Chang, C. Chen, and B. Lee, J. Appl. Phys. 74, 1781 (1993). 